\title{
Papel da Inteligência Emocional, Felicidade e Flow no Desempenho Académico e Bem-Estar Subjetivo em Contexto Universitário
}

\author{
The Role of Emotional Intelligence, Happiness and Flow on Academic Achievement \\ and Subjective Well-Being in the University Context
}

\author{
Helena Alves Moutinho ${ }^{1}$, André Monteiro ${ }^{2}$, Ana Costa ${ }^{3}$ e Luísa Faria ${ }^{4}$
}

\begin{abstract}
Resumo
No quadro da psicologia positiva, pretende-se explorar a relação entre Inteligência Emocional, Felicidade e Flow, e o desempenho académico e bem-estar subjetivo de estudantes universitários. A amostra inclui 215 participantes, com idades entre 18 e 58 anos $(\mathrm{M}=22.3$; $\mathrm{DP}=4.91)$, maioritariamente de Ciências Sociais (55.9\%), de diferentes cursos ( $1 .^{\circ}$ ao $6 .^{\circ}$ ano), e do género feminino (81,9\%), sendo $18,1 \%$ trabalhadoresestudantes. Os instrumentos incluíram escalas de autorrelato (TEIQue, OHI, DFS-2 e JAWS), adaptadas ao contexto português e com bons indicadores psicométricos. Os resultados indicaram correlações positivas entre variáveis positivas e desempenho académico e bem-estar, ainda que menores com o desempenho académico objetivo. Análises de regressão linear demonstraram que o bem-estar subjetivo foi a variável melhor explicada pelas variáveis positivas e que o flow foi a variável com maior poder preditivo sobre o desempenho académico. Serão apresentadas implicações na promoção do desempenho académico e bemestar no contexto universitário.
\end{abstract}

Palavras-chave: inteligência emocional, felicidade, flow, desempenho académico, bem-estar subjetivo

\begin{abstract}
Within the Positive Psychology's framework, this study intends to explore the relationship between Emotional Intelligence, Happiness and Flow, and university students' academic performance and subjective well-being. The sample included 215 participants, aged between 18 and 58 years $(M=22.3 ; S D=4.91)$, from different courses and years $\left(1^{\text {st }}\right.$ to $\left.6^{\text {th }}\right)$, of which $18.1 \%$ were student-workers. Most of the participants were females $(81.9 \%)$, and studied Social Sciences (55.9\%). The instruments included self-report scales (TEIQue, OHI, DFS-2, and JAWS), adapted to the Portuguese context and with good psychometric properties. Results indicated positive correlations between positive variables and academic performance and well-being, although lower correlations with objecticve academic performance. Linear regression analyses showed that subjective well-being was the variable best explained by positive variables and that flow presented the greatest predictive power over academic performance. Implications for the academic performance and wellbeing's promotion in the university context are presented.
\end{abstract}

Keywords: emotional intelligence, happiness, flow, academic performance, subjective well-being

Este trabalho insere-se no projeto "Plataforma Informática Integradora de Aplicações, Serviços Web e Análise de Características Emocionais e de Bem-Estar”, financiado pelo Portugal 2020 (projeto 024012).

\footnotetext{
${ }^{1}$ Bolseira de Iniciação Científica na FPCEUP, no quadro do Projeto 024012. Faculdade de Psicologia e de Ciências da Educação da Universidade do Porto, Portugal. E-mail: helenaiamoutinho@gmail.com

${ }^{2}$ Bolseiro de Iniciação Científica na FPCEUP, no quadro do Projeto 024012. Faculdade de Psicologia e de Ciências da Educação da Universidade do Porto, Portugal. E-mail: andrefmonteiro@outlook.com

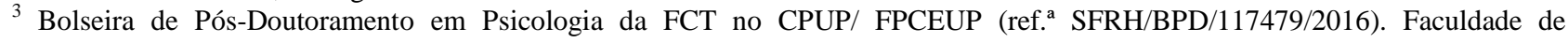
Psicologia e de Ciências da Educação da Universidade do Porto, Portugal. E-mail: ana.fontescosta@ gmail.com

${ }^{4}$ Professora Associada com Agregação da FPCEUP. Faculdade de Psicologia e de Ciências da Educação da Universidade do Porto, Portugal. E-mail: lfaria@ fpce.up.pt

Revista Iberoamericana de Diagnóstico y Evaluación - e Avaliação Psicológica. RIDEP · N52 · Vol.3 · 99-114 · 2019

ISSN: 1135-3848 print /2183-6051online
} 


\section{Introdução}

$\mathrm{Na}$ Psicologia, a perspetiva positiva tem ganho dinamismo em diferentes áreas de intervenção humana (e.g., clínica, educativa e organizacional), quer no contexto de investigação quer no de prática profissional, desde a década de 70 do século XX. Fundada no estudo científico do funcionamento humano ótimo, a psicologia positiva explora quer os fatores que facilitam, quer os que impedem este funcionamento (Linley, Joseph, Harrington, \& Wood, 2006). De facto, esta perspetiva ganhou particular relevo na exploração e compreensão das fontes e processos que levam os indivíduos a atingir os resultados desejados, expresso no interesse pelo estudo da relação entre variáveis positivas (e.g. felicidade, inteligência emocional, afeto positivo, otimismo, flow, autoeficácia, relacionamentos prósperos, entre outras) e resultados nas diversas esferas de realização humana, como o desempenho académico ou profissional, ou indicadores de cariz subjetivo, como os níveis de bem-estar psicológico (Allen \& McCarthy, 2016).

No contexto de trabalho, a literatura tem evidenciado que diferentes variáveis positivas têm demonstrado capacidade de influenciar os resultados dos trabalhadores, fomentando também as suas capacidades de resiliência, por forma a ultrapassar, com sucesso, a adversidade no contexto profissional (Youssef \& Luthans, 2007).

Em particular, no contexto universitário, vários estudos têm vindo a reportar que os jovens se encontram mais sujeitos a diversos fatores de vulnerabilidade relacionados com: a resposta às suas próprias expectativas e às dos seus pais, a deslocalização geográfica, a competição entre pares, as dificuldades em organizar o seu tempo e a preocupação em acabar o curso e ingressar no mercado de trabalho (Silveira, Norton, Brandão, \& Roma-Torres, 2011). Mais ainda, no estudo de Figueira e colaboradores (Figueira, Marques Pinto, Lima, Matos, \& Cherpe, 2014), cerca de um terço dos estudantes em contexto universitário reportavam baixos níveis de saúde mental.

Martin Seligman (2011) defende que o bemestar tem cinco elementos constitutivos: emocionalidade positiva, engagement - entendido como sendo a satisfação sentida mediante o atingimento regular de estados de flow
(Matthewman, Jodhan-Gall, Nowlan, OSullivan, \& Patel, 2018; Seligman, 2011) -, significado, relacionamentos positivos e realizações. Partindo do facto de a influência de variáveis positivas no contexto de realização académica ser ainda pouco explorada, neste estudo consideraremos as variáveis inteligência emocional e felicidade (enquanto integrantes do elemento emocionalidade positiva de Martin Seligman) e o flow (representando o elemento engagement), e a relação destas com o desempenho académico e o bem-estar subjetivo de estudantes universitários, na convicção de que este é um dos contextos de vida mais importantes na preparação para os desafios da vida adulta e do mundo do trabalho.

\section{Inteligência Emocional}

A inteligência emocional (IE) pode ser definida, globalmente, como a capacidade para reconhecer, compreender, expressar e regular emoções (no próprio e nos outros), utilizando, ainda, conteúdo emocional na resolução de problemas (Mayer \& Salovey, 1993). Das várias conceptualizações que surgiram na literatura, Petrides e Furnham (2001) distinguiram dois modelos de IE: (a) a IE como competência mental e (b) a IE como traço de personalidade (Trait Emotional Intelligence-TEI). $\mathrm{O}$ primeiro considera a IE enquanto conjunto de capacidades cognitivo-emocionais, numa perspetiva de medição do desempenho ótimo, enquanto o segundo compreende a IE como um conceito multidimensional, envolvendo autoperceções e disposições afetivas e motivacionais, medindo-se, neste modelo, através do desempenho típico do indivíduo (Perera, 2015).

A literatura tem demonstrado que ambos os modelos de IE se correlacionam significativamente com o desempenho em contexto de trabalho (cf. revisão O'Boyle, Humphrey, Pollack, Hawver, \& Story, 2011). Em particular, o modelo de IE enquanto traço evidencia associações fortes com o desempenho uma vez que partilham componentes importantes, como a consciência, a extroversão ou a estabilidade emocional dos trabalhadores (Barrick, Stewart, \& Piotrowski, 2002; Hurtz \& Donovan, 2000). Mais ainda, a autoeficácia surge como um importante preditor do desempenho uma vez que se traduz na maior motivação, definição 
de objetivos e engagement no trabalho (Erez \& Judge, 2001; Rich, LePine, \& Crawford, 2010). Por outro lado, o modelo de capacidade de IE relaciona-se com o desempenho no trabalho principalmente por promover interações sociais e levar o indivíduo a perceber as exigências emocionais numa determinada situação. Estas competências emocionais (modelo de capacidade de IE) promovem diretamente o desempenho do indivíduo no trabalho, influenciando, também, de forma indireta e positiva, a associação da IE (modelo traço de IE) com o desempenho no trabalho, pelo que apresentam normalmente uma associação forte (Joseph, Jin, Newman, \& O’Boyle, 2015).

No contexto académico, Perera (2016), numa revisão da literatura sobre a relação entre a IEtraço e o desempenho académico, concluiu que a primeira poderá prever o segundo de forma positiva, através de processos cognitivos, motivacionais e interpessoais. Considerando o modelo de competência de IE, a literatura tem evidenciado de forma similar a associação positiva e a capacidade preditiva da IE sobre o rendimento académico dos estudantes (Costa \& Faria, 2014, 2015). Mais recentemente, Bukhari e Khanam (2016) confirmaram esta relação numa amostra de estudantes universitários e Garg, Levin e Tremblay (2016) adiantaram que este efeito parece ser consequência da maior adaptação, que a IE proporciona, do estudante à universidade.

A literatura tem demonstrado também a associação da IE com o bem-estar subjetivo dos indivíduos (Di Fabio \& Kenny, 2016; Koydemir \& Schütz, 2012; Sánchez-Álvarez, Extremera, \& Fernández-Berrocal, 2016; Wang, Zou, Zhang, \& Hou, 2018). Em particular, a IE tende a influenciar o bem-estar subjetivo do indivíduo através da adoção, por este, de estratégias de coping adaptativas para lidar com situações sociais desafiantes, stresse social e conflitos interpessoais, bem como pelo desenvolvimento de redes de suporte social, promovendo a regulação emocional e a vivência de emoções positivas (Zeidner, Matthews, \& Roberts, 2012).

\section{Felicidade}

Lyubomirsky (2008) definiu o conceito de felicidade como um estado mental ou emocional de elevada satisfação, que varia de acordo com a experiência de afetos e emoções positivas, bem como a ausência da experiência de afetos e emoções negativas. A felicidade, para além de depender deste balanço entre a polaridade da experiência afetiva e emocional, também decorre do valor que o indivíduo atribui à sua vida (Lyubomirsky, 2008).

Um outro autor, Diener (1984), por sua vez, refere que a felicidade resulta de uma "propensão geral para se experienciar eventos de forma positiva" (p. 565), fator pelo qual diferentes indivíduos usam diferentes critérios para avaliarem a sua felicidade.

A noção de felicidade e de bem-estar são próximas na literatura, pois o bem-estar consiste num estado de satisfação do indivíduo consigo mesmo e com o seu meio (Ryan \& Deci, 2001). Assim, o bem-estar não é simplesmente a ausência de qualquer psicopatologia, pelo que remete para o funcionamento e experiência psicológica ótima. Emergiram algumas perspetivas teóricas em torno do bem-estar, nomeadamente a de bem-estar psicológico (orientado para a autorrealização do indivíduo, envolvimento no presente e mobilização para o futuro) e a de bem-estar subjetivo, centrado numa perspetiva hedónica e tendo como referência as suas experiências afetivas e emocionais (Ferreira Novo, 2005).

Este constructo compreende uma componente cognitiva - a satisfação com a vida - e uma componente afetiva - referente à experiência de felicidade subjetiva, emoções de prazer e afetos positivos (Diener, Suh, \& Oishi, 1997; Joshnaloo, 2016). Considerando a componente afetiva, Warr (1987, 1990) defende que na perspetiva bidimensional do bem-estar afetivo (segundo a qual diferentes emoções são consideradas em função da intensidade e do prazer que suscitam), existem 3 eixos principais no estudo de bem-estar: (1) desprazer-prazer, (2) ansiedadecontentamento, e (3) depressão-entusiasmo, sendo o segundo e o terceiro eixos de maior importância no domínio ocupacional.

Enquadrando no contexto de trabalho, vários autores verificam uma relação positiva e significativa entre o bem-estar subjetivo e o desempenho laboral, bem como com a criatividade e cidadania organizacional (Jalali \& Heidari, 2016; Man \& Ticu, 2015). Estes resultados corroboram a hipótese de que 
trabalhadores mais felizes conseguem alcançar melhores resultados. Por outro lado, Lyubomirsky, King e Diener (2005) apontam as características da experiência de afeto positivo (e.g. confiança, otimismo e autoeficácia) como promotoras do envolvimento dos indivíduos no alcance de objetivos culturalmente valorizados, enfatizando o papel da felicidade e do bem-estar subjetivo no desempenho humano.

Já no contexto académico, Antaramian (2015) aponta o bem-estar subjetivo, a par da ausência de sintomas psicopatológicos, como sendo um preditor do comprometimento de estudantes universitários. Bukhari e Khanam (2017) mostram também a existência de uma relação positiva e significativa entre $o$ desempenho académico de estudantes universitários, a satisfação com a vida e a felicidade subjetiva, sendo proposto por Lyubomirsky e colaboradores (2005) que esta relação seja reciprocamente fortalecida.

\section{Flow}

Csikszentmihalyi (1990) definiu pela primeira vez na literatura o termo "flow" ou experiência ótima, como um estado de consciência no qual a pessoa que o experiencia está absolutamente envolvida numa atividade, sentindo-se bem pelo simples facto de a desempenhar, e perdendo, inclusive, a noção da passagem do tempo. Csikszentmihalyi (1990) referiu ainda que para alcançar o estado de flow é necessário que o nível de desafio da tarefa e o nível de competência do seu executor sejam percecionados como equilibrados. Segundo o autor, quando o nível de desafio é percebido como elevado e o nível de competência como baixo, o indivíduo entra num estado de ansiedade; e, pelo contrário, quando o nível de desafio é percebido como baixo e o de competência como elevado, o indivíduo entra num estado de aborrecimento (Nakamura \& Csikszentmihalyi, 2002). Mais ainda, quando o desafio da tarefa e o nível de competência são percebidos como baixos, o indivíduo entra num estado de apatia e, por oposição, quando ambos são percebidos como elevados, o indivíduo entra num estado de flow.

Csikszentmihalyi (1990) defende que a experiência de flow se caracteriza por um balanço entre desafio e competência, fusão entre ação e consciência, concentração na tarefa, perda de autoconsciência, transformação do sentido do tempo, clareza de objetivos, perceção de controlo, feedback rigoroso e experiência autotélica, que correspondem às nove dimensões por ele definidas. A primeira, o balanço entre desafio e competência, já referido, está intrinsecamente ligado ao despoletar deste estado nos sujeitos (Delle Fave, 2013). Além disso, a dimensão da fusão entre ação e consciência está relacionada com o facto de, neste estado de consciência, o indivíduo estar absolutamente envolvido no momento presente e naquilo que está a fazer (Csikszentmihalyi, 1990). Este estado de absorção é congruente com uma total concentração na tarefa e perda da autoconsciência, sendo que o indivíduo está de tal forma focado na tarefa que não se preocupa com mais nada, nem mesmo com o seu próprio desempenho na mesma. Com efeito, a absorção na tarefa é tal que a perceção da passagem do tempo é distorcida, parecendo ao indivíduo que este passa mais depressa do que na realidade acontece. Mais ainda, os objetivos são claros para o executor e este percebe-se como tendo controlo sobre aquilo que está a fazer e sobre os resultados que daí advenham, obtendo feedback rigoroso sobre os mesmos, o que significa que o indivíduo sabe, com clareza e em tempo real, se e em que medida está a realizar bem a tarefa. Por fim, todos estes aspetos fazem com que experienciar estados de flow corresponda a uma experiência autotélica (Csikszentmihalyi, 1990), ou seja, a uma experiência que é prazerosa e recompensadora em si mesma, e durante a qual os indivíduos não estão focados nas possíveis consequências do cumprimento da tarefa, mas sim no processo em si.

Na verdade, Csikszentmihalyi (1990) aponta a possibilidade de certos indivíduos terem maior tendência para viverem experiências autotélicas do que outros, afirmando que possuem uma personalidade autotélica. A esta maior predisposição para experimentar estados de flow, independentemente da situação, dá-se o nome de flow-disposicional, por oposição ao flow-estado (Jackson \& Eklund, 2002; Jackson, Ford, Kimiecik, \& Marsh, 1998; Jackson \& Marsh, 1996).

A experiência de flow pode ocorrer em diferentes contextos e atividades, como nas artes 
(Bakker, 2005), no desporto (Gouveia, PaisRibeiro, Marques, \& Carvalho, 2012; Jackson \& Eklund, 2002) ou no trabalho (Ilies et al., 2018). Efetivamente, no contexto laboral, o flow tem um papel importante na absorção dos indivíduos nas tarefas que têm de desempenhar, estando associado a níveis de bem-estar mais elevados (Ilies et al, 2018) e a melhor desempenho (Kuo \& Ho, 2010). No contexto académico, o flow parece estar relacionado com a eficiência quer individual (Sumaya \& Darling, 2018) quer interindividual (Bakker, 2005). Ao nível individual, Sumaya e Darling (2018) verificaram que os estudantes universitários que experienciaram estados de flow durante o desenvolvimento de um trabalho académico tiveram significativamente melhores resultados do que aqueles que não $o$ experienciaram. Também Joo, Oh e Kim (2015), por seu lado, encontraram uma relação de predição do flow sobre o desempenho académico, de $16,5 \%$, em estudantes de uma universidade online, na Coreia. Em termos interindividuais, Bakker (2005) verificou que, em momentos letivos, alunos de música experienciavam tanto mais estados de flow quanto mais os seus professores os experienciavam, o que sugere o efeito de contágio na experiência de flow.

A literatura tem também demonstrado que o flow é promotor do bem-estar de estudantes universitários. Asakawa (2004) verificou que, no Japão, estudantes universitários autotélicos (i.e., alunos que reportavam altos níveis de flow disposicional) obtinham níveis mais elevados de Jujitsu-kan, que corresponde a um sentimento de elevada realização e satisfação com a vida. Steele e Fullagar (2009), por sua vez, encontraram um impacto positivo do flow sobre o bem-estar físico e psicológico de estudantes universitários.

Neste quadro, o objetivo deste estudo é o de explorar a relação entre variáveis positivas inteligência emocional, felicidade e flow -, desempenho académico e bem-estar subjetivo em estudantes universitários. Em particular, é esperado que as variáveis positivas estabeleçam associações positivas com os resultados académicos e bem-estar do estudante. Mais ainda, a IE, felicidade e flow deverão individualmente ter capacidade de predizer os resultados académicos dos estudantes e o seu bem-estar, ainda que possam ter maior capacidade preditiva sobre os resultados de cariz subjetivo (desempenho académico subjetivo e bem-estar subjetivo) do que sobre o seu desempenho objetivo.

\section{Método}

\section{Participantes}

A amostra inclui 215 estudantes $(81.9 \%$ do género feminino), com uma média etária de 22 anos $(M=22.3 ; D P=4.91)$, a frequentar diferentes cursos universitários, maioritariamente de Ciências Sociais $(55.9 \%)$. A maioria dos participantes frequenta o $2 .^{\circ}$ ano de licenciatura $(35.8 \%)$ e $18.1 \%$ são trabalhadores-estudantes.

\section{Instrumentos}

\section{Inteligência emocional}

Para avaliar a IE utilizou-se o TEIQue-SF (Trait-Emotional Intelligence Questionnaire Short Version), desenvolvido por Petrides e Furnham (2006) e adaptado para a população portuguesa por Carvalho, Neto e Mavroveli (2010). Este questionário de autorrelato compreende 4 dimensões: emocionalidade (e.g., "Exprimir as minhas emoções com palavras não é um problema para mim."), sociabilidade (e.g., "Consigo lidar eficazmente com as pessoas."), autocontrolo (e.g., "Tendo a mudar de ideias frequentemente.") e bem-estar (e.g., "Geralmente não acho a vida agradável.") e é constituído por 30 itens com uma escala de resposta de Likert de 7 pontos ("1-Discordo totalmente" a "7-Concordo totalmente"). Segundo vários autores (Carvalho et al., 2010; Petrides \& Furnham, 2006), o TEIQue-SF apresenta boas qualidades psicométricas, providenciando resultados fiáveis, e cuja estrutura de 4 dimensões é suportada por estudos de análise fatorial. Neste estudo, a análise fatorial exploratória (EFA) evidenciou os 4 fatores propostos, que apresentavam $51.3 \%$ de variância total explicada. Os valores de comunalidade foram na generalidade superiores a .40 , sendo que os pesos fatoriais ou saturações foram superiores a .50. No que se refere à consistência interna, a escala total apresentou um valor alfa de Cronbach excelente $(\alpha=.90)$.

\section{Felicidade}

$\mathrm{O}$ Oxford Happiness Inventory (OHI), desenvolvido por Argyle, Martin e Crossland 
(1989), foi adaptado ao contexto português por Neto (2001), e adotado neste estudo para avaliar o constructo felicidade. Este instrumento de autorrelato é constituído por 29 itens e compreende 7 dimensões: alegria, eficácia pessoal, bem-estar físico, perspetiva positiva, satisfação com a vida, autoestima e sociabilidade/empatia, sendo apresentadas, em cada item, 4 afirmações distintas numa escala de 4 pontos, entre 0 e 3 (e.g. sociabilidade/empatia, "0 - Eu passo pela vida", "1 - A vida é boa", "2 - A vida é muito boa" e "3 - Eu amo a vida"). $\mathrm{O}$ participante deve assinalar a opção de resposta que mais se aproxime da forma como se tem sentido na última semana, incluindo no próprio dia.

No presente estudo, a AFE confirmou as 7 dimensões teóricas do instrumento, que explicavam $63 \%$ de variância, com valores de comunalidade e pesos fatoriais ou saturações acima de .42 e .50 , respetivamente. $\mathrm{O}$ instrumento revelou ainda níveis excelentes de consistência interna para a escala total $(\alpha=.93)$.

\section{Flow}

Para avaliar o flow-disposicional foi utilizada a Dispositional Flow Scale-2 (DFS-2), desenvolvida por Jackson e Eklund (2002) e adaptada ao contexto português por Gouveia, Pais-Ribeiro, Marques e Carvalho (2012). Esta escala de autorrelato foi originalmente desenvolvida para avaliar a tendência de um indivíduo para experienciar estados de flow durante a atividade física, sendo que, neste estudo, as referências à atividade física foram adaptadas para referências a atividades académicas. A versão portuguesa da escala é constituída por 43 itens, mas neste estudo foram adotados apenas os 36 itens da versão original do instrumento. A escala avalia as 9 dimensões propostas por Csikszentmihalyi (1990): balanço entre desafio e competência (e.g., "Sinto-me desafiado(a) mas acredito que as minhas capacidades estão à altura das exigências da situação."), fusão entre ação e consciência, clareza de objetivos, feedback rigoroso, concentração na tarefa, perceção de controlo, perda da autoconsciência, transformação do sentido de tempo e experiência autotélica. A versão portuguesa adaptada do instrumento apresentou globalmente qualidades psicométricas adequadas (Gouveia et al., 2012). Neste estudo, a
AFE confirmou as 9 dimensões propostas teoricamente, que conjuntamente explicavam $73.4 \%$ da variância total dos dados. Globalmente, o instrumento apresentou comunalidades superiores a .59 e pesos fatoriais ou saturações superiores a .54. Mais ainda, o instrumento revelou excelentes níveis de consistência interna $(\alpha=.94)$.

\section{Bem-estar subjetivo}

A escala Job-related Affective Well-being Scale (JAWS) foi desenvolvida por Katwyk, Fox, Spector e Kelloway (2000), baseando-se no modelo de bem-estar afetivo no trabalho de Warr (1987). Este instrumento foi adaptado para a língua portuguesa, no Brasil, por Gouveia, Fonsêca, Lins, Lima e Gouveia (2008), tendo sido esta a versão utilizada neste estudo. Na sua versão curta, são apresentados 12 itens (12 emoções - 6 negativas (e.g., "Incomodado(a)") e 6 positivas (e.g., "Entusiasmado(a)") com uma escala de resposta de frequência de tipo Likert de 5 pontos ("1 - Nunca" a "5 - Sempre"), relativamente às emoções sentidas enquanto realizavam atividades relacionadas com o seu desempenho académico, nos últimos 30 dias. No presente estudo, a AFE revelou a existência dos dois fatores (afeto positivo e afeto negativo) identificados no estudo prévio em contexto português (Gouveia et al., 2008), que explicam $67.2 \%$ de variância total dos dados. Os itens apresentaram valores adequados de comunalidade e pesos fatoriais ou saturações (superiores a .54 e .70 , respetivamente). No que respeita à consistência interna, a escala total $(\alpha=.92)$, a subescala positiva $(\alpha=.90)$ e a subescala negativa $(\alpha=.88)$ apresentaram bons índices.

\section{Desempenho académico}

O desempenho académico foi avaliado segundo dois indicadores:

Desempenho académico subjetivo - foi avaliada a perceção do sujeito sobre a sua competência académica, neste caso, "como se considerava enquanto aluno/a", segundo uma escala de resposta de tipo Likert de 5 pontos ("1 Mau (má)" a "5 - Excelente"). A amplitude de respostas dos participantes variou entre 1 e 5 $(M=2.87 ; D P=.83)$.

Desempenho académico objetivo - por sua vez, a avaliação objetiva foi obtida através da 
resposta dos participantes acerca do valor da sua média académica atual. As respostas foram compreendidas entre 10.5 e 20 valores $(M=14.5$; $D P=1.83)$.

\section{Procedimento}

A recolha de dados foi realizada por questionário, que incluiu os instrumentos acima referidos, perguntas de natureza sociodemográfica, bem como algumas questões acerca do percurso académico do estudante. A administração deste questionário decorreu online, através da submissão de respostas na plataforma Google Forms, a uma amostra de conveniência (i.e., redes pessoais dos investigadores, divulgação a estudantes universitários por parte de professores e divulgação em redes sociais). $\mathrm{O}$ objetivo do presente estudo foi apresentado aos participantes e o consentimento informado assegurado no início da participação, sendo dada aos participantes a opção de desistir a qualquer momento e sem que houvesse quaisquer consequências. O tempo de resposta foi, em média, de vinte minutos. A análise de dados (i.e., correlações, regressões, análises fatoriais exploratórias e alfa de Cronbach) foi efetuada com recurso ao programa IBM SPSS Statistics (Versão 25.0).

\section{Resultados}

Globalmente, as análises correlacionais evidenciaram uma relação positiva e significativa entre as variáveis positivas (IE, felicidade e flow) e os resultados académicos dos estudantes e o seu bem-estar subjetivo (cf. Quadro 1). Os resultados demonstraram que as variáveis positivas atingiam maior magnitude na relação com o bem-estar subjetivo do estudante, seguido pela perceção subjetiva de desempenho do estudante, e, com menor intensidade, com o desempenho objetivo do estudante. Das variáveis em estudo, as dimensões de autocontrolo e bem-estar da IE, de bem-estar físico, perspetiva positiva e satisfação com a vida (felicidade), e experiência autotélica e balanço entre desafio e competência (flow) foram as que se correlacionaram, com magnitudes mais elevadas, com o desempenho académico dos estudantes e com o seu bem-estar subjetivo.

No sentido de explorar o poder preditivo das variáveis positivas sobre o desempenho académico (objetivo e subjetivo) e o bem-estar dos estudantes, foram realizadas análises de regressão linear. Verificou-se que os modelos explicativos dos resultados académicos (i.e., desempenho académico objetivo, subjetivo e bemestar subjetivo), preditos pelas variáveis positivas, foram significativos (cf. Quadros 2, 3 e 4). O poder preditivo das variáveis positivas foi mais evidente sobre o bem-estar subjetivo dos estudantes ( $R^{2}$ ajust. 59\%; cf. Quadro 4), e sobre a autoavaliação dos estudantes (i.e., desempenho subjetivo; $R^{2}$ ajust. 28\%; cf. Quadro 3), sendo o desempenho académico objetivo dos estudantes (i.e., média das notas) o modelo menos explicado pelas variáveis em estudo ( $R^{2}$ ajust. $13 \%$; cf. Quadro 2). Os resultados demonstraram que o flow foi a única variável que demonstrou capacidade preditiva em todos os modelos explicativos.

\section{Discussão}

Em contextos de existência progressivamente mais competitivos, a perspetiva da psicologia positiva sobre o funcionamento humano tem ganho dinamismo, uma vez que explora os processos através dos quais os indivíduos, em resposta às exigências do meio, atingem resultados desejáveis (Allen \& McCarthy, 2016).

Neste quadro, este estudo procurou explorar a importância que variáveis positivas como a inteligência emocional, a felicidade e o flow têm sobre o desempenho académico de estudantes do ensino universitário, quer considerando o desempenho objetivo (i.e., média de notas) quer subjetivo (i.e., perceção de competência do estudante), quer o seu bem-estar subjetivo.

Globalmente, os resultados evidenciaram correlações positivas, de magnitude média a forte, entre as variáveis positivas e os resultados académicos e bem-estar, corroborando os resultados da literatura (Asakawa, 2004; Bukhari \& Khanam, 2017; Di Fabio \& Kenny, 2016; Lyubomirsky et al., 
Quadro 1. Correlações de Pearson entre variáveis positivas, desempenho académico e bem-estar subjetivo

\begin{tabular}{|c|c|c|c|c|c|c|c|c|c|c|c|c|c|c|}
\hline & 1. & 2. & 3. & 4. & 5. & 6. & 7. & 8. & 9. & 10. & 11. & 12. & 13. & 14. \\
\hline $\begin{array}{l}\text { 1. Inteligência } \\
\text { Emocional total }\end{array}$ & 1 & & & & & & & & & & & & & \\
\hline 2. Emocionalidade & $.78^{* *}$ & 1 & & & & & & & & & & & & \\
\hline 3. Sociabilidade & $.74^{* *}$ & $.51^{* *}$ & 1 & & & & & & & & & & & \\
\hline 4. Autocontrolo & $.74^{* *}$ & $.43^{* *}$ & $.42^{* *}$ & 1 & & & & & & & & & & \\
\hline 5. Bem-estar & $.86^{* *}$ & $.55^{* *}$ & $.58^{* *}$ & $.52^{* *}$ & 1 & & & & & & & & & \\
\hline 6. Felicidade total & $.80^{* *}$ & $.54^{* *}$ & $.53^{* *}$ & $.51^{* *}$ & $.82^{* *}$ & 1 & & & & & & & & \\
\hline 7. Alegria & $.64^{* *}$ & $.47^{* *}$ & $.43^{* *}$ & $.38^{* *}$ & $.67^{* *}$ & $.79^{* *}$ & 1 & & & & & & & \\
\hline 8. Eficácia pessoal & $.68^{* *}$ & $.43^{* *}$ & $.50^{* *}$ & $.45^{* *}$ & $.71^{* *}$ & $.86^{* *}$ & $.62^{* *}$ & 1 & & & & & & \\
\hline 9. Bem-estar físico & $.71^{* *}$ & $.49^{* *}$ & $.47^{* *}$ & $.48^{* *}$ & $.70^{* *}$ & $.85^{* *}$ & $.62^{* *}$ & $.69^{* *}$ & 1 & & & & & \\
\hline $\begin{array}{l}\text { 10. Perspetiva } \\
\text { positiva }\end{array}$ & $.69^{* *}$ & $.48^{* *}$ & $.45^{* *}$ & $.48^{* *}$ & $.67^{* *}$ & $.87^{* *}$ & $.63^{* *}$ & $.72^{* *}$ & $.69^{* *}$ & 1 & & & & \\
\hline $\begin{array}{l}\text { 11. Satisfação com a } \\
\text { vida }\end{array}$ & $.66^{* *}$ & $.44^{* *}$ & $.45^{* *}$ & $.40^{* *}$ & $.70^{* *}$ & $.87^{* *}$ & $.69^{* *}$ & $.65^{* *}$ & $.71^{* *}$ & $.71^{* *}$ & 1 & & & \\
\hline 12. Autoestima & $.59^{* *}$ & $.38^{* *}$ & $.38^{* *}$ & $.40^{* *}$ & $.61^{* *}$ & $.74^{* *}$ & $.49^{* *}$ & $.64^{* *}$ & $.60^{* *}$ & $.62^{* *}$ & $.55^{* *}$ & 1 & & \\
\hline $\begin{array}{l}13 . \\
\text { Sociabilidade/Empatia }\end{array}$ & $.76^{* *}$ & $.53^{* *}$ & $.48^{* *}$ & $.46^{* *}$ & $.80^{* *}$ & $.91^{* *}$ & $.73^{* *}$ & $.75^{* *}$ & $.76^{* *}$ & $.73^{* *}$ & $.75^{* *}$ & $.64^{* *}$ & 1 & \\
\hline 14. Flow total & $.56^{* *}$ & $.35^{* *}$ & $.42^{* *}$ & $.36^{* *}$ & $.52^{* *}$ & $.67^{* *}$ & $.48^{* *}$ & $.56^{* *}$ & $.50^{* *}$ & $.61^{* *}$ & $.57^{* *}$ & $.49^{* *}$ & $.61^{* *}$ & 1 \\
\hline $\begin{array}{l}\text { 15. Fusão entre ação e } \\
\text { consciência }\end{array}$ & $.21^{* *}$ & .011 & $.25^{* *}$ & $.17^{*}$ & $.19^{* *}$ & $.31^{* *}$ & $.17^{*}$ & $.28^{* *}$ & $.21^{*}$ & $.34^{* *}$ & $.28^{* *}$ & $.26^{* *}$ & $.25^{* *}$ & $.69^{* *}$ \\
\hline $\begin{array}{l}\text { 16. Experiência } \\
\text { autotélica }\end{array}$ & $.45^{* *}$ & $.25^{* *}$ & $.26^{* *}$ & $.30^{* *}$ & $.47^{* *}$ & $.60^{* *}$ & $.42^{* *}$ & $.50^{* *}$ & $.55^{* *}$ & $.56^{* *}$ & $.51^{* *}$ & $.44^{* *}$ & $.54^{* *}$ & $.81^{* *}$ \\
\hline $\begin{array}{l}\text { 17. Balanço entre } \\
\text { desafio e competência }\end{array}$ & $.54^{* *}$ & $.28^{* *}$ & $.34^{* *}$ & $.39^{* *}$ & $.55^{* *}$ & $.63^{* *}$ & $.46^{* *}$ & $.48^{* *}$ & $.61^{* *}$ & $.60^{* *}$ & $.54^{* *}$ & $.47^{* *}$ & $.57^{* *}$ & $.84^{* *}$ \\
\hline $\begin{array}{l}\text { 18. Clareza de } \\
\text { objetivos }\end{array}$ & $.55^{* *}$ & $.43^{* *}$ & $.36^{* *}$ & $.34^{* *}$ & $.51^{* *}$ & $.59^{* *}$ & $.41^{* *}$ & $.49^{* *}$ & $.54^{* *}$ & $.52^{* *}$ & $.48^{* *}$ & $.46^{* *}$ & $.58^{* *}$ & $.78^{* *}$ \\
\hline $\begin{array}{l}\text { 19. Concentração na } \\
\text { tarefa }\end{array}$ & $.38^{* *}$ & $.28^{* *}$ & $.27^{* *}$ & $.23^{* *}$ & $.32^{* *}$ & $.46^{* *}$ & $.32^{* *}$ & $.40^{* *}$ & $.44^{* *}$ & $.40^{* *}$ & $.44^{* *}$ & $.31^{* *}$ & $.40^{* *}$ & $.76^{* *}$ \\
\hline $\begin{array}{l}\text { 20. Perda de } \\
\text { autoconsciência }\end{array}$ & $.26^{* *}$ & $.20^{* *}$ & $.28^{* *}$ & $.17^{*}$ & $.20^{* *}$ & $.27^{* *}$ & $.20^{* *}$ & $.25^{* *}$ & $.17^{*}$ & $.23^{* *}$ & $.24^{* *}$ & $.20^{* *}$ & $.28^{* *}$ & $.43^{* *}$ \\
\hline $\begin{array}{l}\text { 21. Perceção de } \\
\text { controlo }\end{array}$ & $.48^{* *}$ & $.26^{* *}$ & $.38^{* *}$ & $.32^{* *}$ & $.45^{* *}$ & $.58^{* *}$ & $.42^{* *}$ & $.45^{* *}$ & $.54^{* *}$ & $.54^{* *}$ & $.52^{* *}$ & $.40^{* *}$ & $.51^{* *}$ & $.81^{* *}$ \\
\hline $\begin{array}{l}\text { 22. Transformação do } \\
\text { sentido de tempo }\end{array}$ & .051 & .090 & .13 & $-.14^{*}$ & .054 & $.14^{*}$ & $.17^{*}$ & $.17^{*}$ & .12 & .098 & .08 & .108 & $.14^{*}$ & $.36^{* *}$ \\
\hline 23. Feedback rigoroso & $.57^{* *}$ & $.35^{* *}$ & $.38^{* *}$ & $.47^{* *}$ & $.52^{* *}$ & $.60^{* *}$ & $.42^{* *}$ & $.50^{* *}$ & $.57^{* *}$ & $.54^{* *}$ & $.50^{* *}$ & $.43^{* *}$ & $.55^{* *}$ & $.85^{* *}$ \\
\hline $\begin{array}{l}\text { 24. Desempenho } \\
\text { académico objetivo }\end{array}$ & $.19^{* *}$ & .059 & .12 & $.21^{* *}$ & $.16^{*}$ & $.19^{* *}$ & .12 & $.17^{*}$ & $.28^{* *}$ & $.16^{*}$ & $.18^{* *}$ & .036 & $.15^{*}$ & $.23^{* *}$ \\
\hline $\begin{array}{l}\text { 25. Desempenho } \\
\text { académico subjetivo }\end{array}$ & $.35^{* *}$ & $.16^{*}$ & $.20^{* *}$ & $.31^{* *}$ & $.34^{* *}$ & $.40^{* *}$ & $.24^{* *}$ & $.32^{* *}$ & $.41^{* *}$ & $.38^{* *}$ & $.35^{* *}$ & $.27^{* *}$ & $.35^{* *}$ & $.45^{* *}$ \\
\hline $\begin{array}{l}\text { 26. Bem-estar } \\
\text { Subjetivo total }\end{array}$ & $.51^{* *}$ & $.28^{* *}$ & $.30^{* *}$ & $.42^{* *}$ & $.49^{* *}$ & $.56^{* *}$ & $.38^{* *}$ & $.47^{* *}$ & $.53^{* *}$ & $.55^{* *}$ & $.51^{* *}$ & $.37^{* *}$ & $.48^{* *}$ & $.64^{* *}$ \\
\hline 27. Afeto positivo & $.51^{* *}$ & $.28^{* *}$ & $.30^{* *}$ & $.40^{* *}$ & $.50^{* *}$ & $.62^{* *}$ & $.42^{* *}$ & $.55^{* *}$ & $.56^{* *}$ & $.60^{* *}$ & $.53^{* *}$ & $.46^{* *}$ & $.54^{* *}$ & $.71^{* *}$ \\
\hline 28. Afeto negativo & $-.42^{* *}$ & $-.23^{* *}$ & $-.24^{* *}$ & $-.36^{* *}$ & $-.39^{* *}$ & $-.40^{* *}$ & $-.26^{* *}$ & $-.31^{* *}$ & $-.39^{* *}$ & $-.40^{* *}$ & $-.39^{* *}$ & $-.22^{* *}$ & $-.34^{* *}$ & $-.44^{* *}$ \\
\hline
\end{tabular}

2005; Perera, 2016; Sumaya \& Darling, 2018).

Esta associação foi mais elevada entre as variáveis positivas e o desempenho académico subjetivo (bem-estar subjetivo, seguido de desempenho subjetivo), o que reforça a importância, já retratada na literatura, de competências cognitivoemocionais na perceção de bem-estar dos estudantes (Di Fabio \& Kenny, 2016; Koydemir \& Schütz, 2012; Sánchez-Álvarez et al., 2016; Steele \& Fullagar, 2009; Wang et al., 2018). Mais ainda, enquanto todas as dimensões de IE, felicidade e flow (à exceção da dimensão da transformação do sentido de tempo) se correlacionaram com as perceções dos estudantes (quer de bem-estar quer de desempenho académico), apenas algumas demonstraram correlacionar-se com o desempenho objetivo (i.e., média das notas). Em particular, o autocontrolo e o bem-estar correlacionaram-se com o desempenho académico objetivo, corroborando a literatura que sustenta a associação entre IE e rendimento académico (Bukhari \& Khanam, 2016; Costa \& Faria, 2014, 2015; O'Boyle et al., 2011; Perera, 2016). Também a felicidade, através das dimensões de bem-estar físico, eficácia pessoal, perspetiva positiva, satisfação com a vida e sociabilidade/empatia, se associou positivamente com o desempenho académico, tal como foi evidenciado em estudos anteriores (Bukhari \& Khanam, 2017; Lyubomirsky et al., 2005). À exceção das dimensões perda da autoconsciência e transformação do sentido de tempo, todas as dimensões 
Quadro 1. Correlações de Pearson entre variáveis positivas, desempenho académico e bem-estar subjetivo

\begin{tabular}{|c|c|c|c|c|c|c|c|c|c|c|c|c|c|c|}
\hline \multicolumn{15}{|c|}{ (Cont.) } \\
\hline & 15. & 16. & 17. & 18. & 19. & 20. & 21. & 22. & 23. & 24. & 25. & 26. & 27. & 28. \\
\hline $\begin{array}{l}\text { 1. Inteligência } \\
\text { Emocional total }\end{array}$ & & & & & & & & & & & & & & \\
\hline 2. Emocionalidade & & & & & & & & & & & & & & \\
\hline 3. Sociabilidade & & & & & & & & & & & & & & \\
\hline 4. Autocontrolo & & & & & & & & & & & & & & \\
\hline 5. Bem-estar & & & & & & & & & & & & & & \\
\hline 6. Felicidade total & & & & & & & & & & & & & & \\
\hline 7. Alegria & & & & & & & & & & & & & & \\
\hline 8. Eficácia pessoal & & & & & & & & & & & & & & \\
\hline 9. Bem-estar físico & & & & & & & & & & & & & & \\
\hline $\begin{array}{l}\text { 10. Perspetiva } \\
\text { positiva } \\
\text { 11. Satisfação com a } \\
\text { vida }\end{array}$ & & & & & & & & & & & & & & \\
\hline 12. Autoestima & & & & & & & & & & & & & & \\
\hline $\begin{array}{l}13 . \\
\text { Sociabilidade/Empatia }\end{array}$ & & & & & & & & & & & & & & \\
\hline 14. Flow total & & & & & & & & & & & & & & \\
\hline $\begin{array}{l}\text { 15. Fusão entre ação e } \\
\text { consciência }\end{array}$ & 1 & & & & & & & & & & & & & \\
\hline $\begin{array}{l}\text { 16. Experiência } \\
\text { autotélica }\end{array}$ & $.47^{* *}$ & 1 & & & & & & & & & & & & \\
\hline $\begin{array}{l}\text { 17. Balanço entre } \\
\text { desafio e competência }\end{array}$ & $.54^{* *}$ & $.75^{* *}$ & 1 & & & & & & & & & & & \\
\hline $\begin{array}{l}\text { 18. Clareza de } \\
\text { objetivos }\end{array}$ & $.38^{* *}$ & $.65^{* *}$ & $.64^{* *}$ & 1 & & & & & & & & & & \\
\hline $\begin{array}{l}\text { 19. Concentração na } \\
\text { tarefa }\end{array}$ & $.46^{* *}$ & $.66^{* *}$ & $.58^{* *}$ & $.52^{* *}$ & 1 & & & & & & & & & \\
\hline $\begin{array}{l}\text { 20. Perda de } \\
\text { autoconsciência }\end{array}$ & $.30^{* *}$ & .073 & $.17^{*}$ & $.23^{* *}$ & $.21^{* *}$ & 1 & & & & & & & & \\
\hline $\begin{array}{l}\text { 21. Perceção de } \\
\text { controlo }\end{array}$ & $.59^{* *}$ & $.57^{* *}$ & $.71^{* *}$ & $.54^{* *}$ & $.66^{* *}$ & $.30^{* *}$ & 1 & & & & & & & \\
\hline $\begin{array}{l}\text { 22. Transformação do } \\
\text { sentido de tempo }\end{array}$ & $.18^{* *}$ & $.27^{* *}$ & .13 & $.20^{* *}$ & $.22^{* *}$ & .043 & .071 & 1 & & & & & & \\
\hline 23. Feedback rigoroso & $.52^{* *}$ & $.68^{* *}$ & $.77^{* *}$ & $.68^{* *}$ & $.60^{* *}$ & $.27^{* *}$ & $.71^{* *}$ & $.16^{*}$ & 1 & & & & & \\
\hline $\begin{array}{l}\text { 24. Desempenho } \\
\text { académico objetivo }\end{array}$ & .093 & $.32^{* *}$ & $.31^{* *}$ & $.14^{*}$ & $.18^{* *}$ & -.058 & $.22^{* *}$ & .028 & $.26^{* *}$ & 1 & & & & \\
\hline $\begin{array}{l}\text { 25. Desempenho } \\
\text { académico subjetivo }\end{array}$ & $.24^{* *}$ & $.50^{* *}$ & $.53^{* *}$ & $.39^{* *}$ & $.34^{* *}$ & -.015 & $.38^{* *}$ & .046 & $.48^{* *}$ & $.72^{* *}$ & 1 & & & \\
\hline $\begin{array}{l}\text { 26. Bem-estar } \\
\text { Subjetivo total }\end{array}$ & $.29^{* *}$ & $.73^{* *}$ & $.63^{* *}$ & $.51^{* *}$ & $.48^{* *}$ & $.17^{*}$ & $.52^{* *}$ & .088 & $.59^{* *}$ & $.35^{* *}$ & $.40^{* *}$ & 1 & & \\
\hline 27. Afeto positivo & $.39^{* *}$ & $.81^{* *}$ & $.68^{* *}$ & $.58^{* *}$ & $.56^{* *}$ & .13 & $.53^{* *}$ & $.21^{* *}$ & $.61^{* *}$ & $.33^{* *}$ & $.44^{* *}$ & $.89^{* *}$ & 1 & \\
\hline 28. Afeto negativo & $-.15^{*}$ & $-.52^{* *}$ & $-.47^{* *}$ & $-.34^{* *}$ & $-.31^{* *}$ & $-.17^{*}$ & $-.40^{* *}$ & .038 & $-.46^{* *}$ & $-.30^{* *}$ & $-.28^{* *}$ & $-.91^{* *}$ & $-.62^{* *}$ & 1 \\
\hline
\end{tabular}

do flow revelaram estar significativamente relacionadas com a média das notas, confirmando a relevância do flow no desempenho académico (Joo et al., 2015; Sumaya \& Darling, 2018). O facto de estas duas dimensões não se correlacionarem com o desempenho objetivo poderá dever-se a limitações metodológicas, já encontradas por Gouveia e colaboradores (2012). Desta forma, os recursos socioemocionais dos estudantes, a par da sua perceção de controlo, parecem demonstrar particular relevância no seu desempenho em contexto universitário, na medida em que lhes permitem adaptar-se às exigências do meio académico.
Os resultados obtidos neste estudo evidenciaram ainda o poder preditivo das variáveis positivas sobre o desempenho académico e o bem-estar. Globalmente, verificouse que os modelos explicativos do desempenho académico (i.e., desempenho objetivo e subjetivo e bem-estar do estudante) foram significativamente preditos pelas variáveis positivas. Em particular, os resultados da análise de regressão linear evidenciaram, a par da maior correlação verificada neste estudo, que o bemestar subjetivo e o desempenho académico subjetivo foram os modelos mais explicados pelas variáveis positivas ( $R^{2}$ ajustado $59 \%$ e $28 \%$, respetivamente). 
Quadro 2. Modelos de regressão linear de variáveis positivas sobre o desempenho académico objetivo

\begin{tabular}{|c|c|c|c|c|c|c|c|c|}
\hline Modelo & & $t$ & $p$ & $\beta$ & $F$ & $g l$ & $p$ & $\begin{array}{c}R^{2} \\
\text { ajustado }\end{array}$ \\
\hline \multirow[t]{24}{*}{$\begin{array}{l}\text { Desempenho } \\
\text { académico } \\
\text { objetivo }\end{array}$} & & & & & 2.66 & 20 & .001 & .13 \\
\hline & $\begin{array}{l}\text { Inteligência emocional } \\
\text { (TEIQue-SF) }\end{array}$ & & & & & & & \\
\hline & Emocionalidade & -.38 & .70 & -.034 & & & & \\
\hline & Sociabilidade & .74 & .46 & .064 & & & & \\
\hline & Autocontrolo & 1.55 & .12 & .13 & & & & \\
\hline & Bem-estar & -.58 & .57 & -.074 & & & & \\
\hline & Felicidade (OHI) & & & & & & & \\
\hline & Alegria & -.71 & .48 & -.072 & & & & \\
\hline & Eficácia pessoal & .73 & .46 & .085 & & & & \\
\hline & Bem-estar físico & 2.06 & .041 & .24 & & & & \\
\hline & Perspetiva positiva & -.82 & .41 & -.095 & & & & \\
\hline & Satisfação com a vida & .49 & .63 & .056 & & & & \\
\hline & Autoestima & -2.33 & .021 & -.21 & & & & \\
\hline & Sociabilidade/empatia & -.46 & .65 & -.065 & & & & \\
\hline & Flow (DFS-2) & & & & & & & \\
\hline & Fusão entre ação e consciência & -1.16 & .25 & -.11 & & & & \\
\hline & Experiência autotélica & 2.79 & .006 & .34 & & & & \\
\hline & $\begin{array}{c}\text { Balanço entre desafio e } \\
\text { competência }\end{array}$ & 1,33 & .19 & .17 & & & & \\
\hline & Clareza de objetivos & -1.54 & .13 & -.15 & & & & \\
\hline & Concentração na tarefa & -1.12 & .26 & -.11 & & & & \\
\hline & Perda de autoconsciência & -.78 & .44 & -.057 & & & & \\
\hline & Perceção de controlo & .70 & .48 & .082 & & & & \\
\hline & $\begin{array}{l}\text { Transformação do sentido de } \\
\text { tempo }\end{array}$ & .009 & .99 & .001 & & & & \\
\hline & Feedback rigoroso & .41 & .68 & .051 & & & & \\
\hline
\end{tabular}

Nota. Os valores $p$ a negrito são estatisticamente significativos.

De facto, uma vez que as variáveis positivas preditoras são avaliadas subjetivamente segundo medidas de autorrelato, torna-se plausível que possam demonstrar maior poder explicativo em modelos com indicadores iminentemente subjetivos do aluno, como o bem-estar e o desempenho académico subjetivo, quando comparados com modelos com indicadores mais objetivos, como, por exemplo, o modelo da média das notas dos alunos.

As análises de regressão linear demonstraram que o flow foi a variável com maior poder preditivo sobre o desempenho académico em todos os modelos. De facto, a avaliação do estado de flow é eminentemente disposicional e dependente do contexto a que se aplica (neste caso, o académico), o que conduz a um maior poder preditivo desta variável sobre o desempenho académico, quando comparada com as outras variáveis positivas (IE e felicidade), de avaliação mais generalizada e independente da especificidade do contexto. No entanto, o poder preditivo do flow (Asakawa, 2004; Bakker, 2005; Joo et al., 2015; Sumaya \& Darling, 2018), confirmado pelos resultados encontrados, revela a pertinência do estudo desta variável em contextos em que o desempenho é valorizado.

Por outro lado, o bem-estar dos estudantes foi essencialmente predito pelo estado de flow e autocontrolo (IE). De facto, a sensação de flow equivalente a um estado pleno e fluído de funcionamento positivo, em que, por um lado, o indivíduo domina a execução da tarefa, e, por outro, consegue envolver-se totalmente nesta experiência - torna-se prazerosa e recompensadora em si mesma e pressupõe a sensação de bem-estar do indivíduo. Da mesma forma, a sensação de que o indivíduo está em controlo traduz-se na presença de afetos positivos, o que reforça a sensação positiva de bem-estar. 
Quadro 3. Modelos de regressão linear de variáveis positivas sobre o desempenho académico subjetivo.

\begin{tabular}{|c|c|c|c|c|c|c|c|c|}
\hline Modelo & & $t$ & $p$ & $\beta$ & $F$ & $g l$ & $p$ & $\begin{array}{c}R^{2} \\
\text { ajustado }\end{array}$ \\
\hline \multirow{24}{*}{$\begin{array}{l}\text { Desempenho } \\
\text { académico } \\
\text { subjetivo }\end{array}$} & & & & & 5.21 & 20 & .001 & .28 \\
\hline & $\begin{array}{c}\text { Inteligência emocional (TEIQue } \\
\text { SF) }\end{array}$ & & & & & & & \\
\hline & Emocionalidade & -.94 & .35 & -.076 & & & & \\
\hline & Sociabilidade & .30 & .76 & .024 & & & & \\
\hline & Autocontrolo & 1.11 & .27 & .086 & & & & \\
\hline & Bem-estar & -.011 & .991 & -.001 & & & & \\
\hline & Felicidade (OHI) & & & & & & & \\
\hline & Alegria & -1.06 & .29 & -.098 & & & & \\
\hline & Eficácia pessoal & -.011 & .998 & $<.001$ & & & & \\
\hline & Bem-estar físico & .93 & .35 & .099 & & & & \\
\hline & Perspetiva positiva & .46 & .64 & .049 & & & & \\
\hline & Satisfação com a vida & .69 & .49 & .071 & & & & \\
\hline & Autoestima & -.48 & .63 & -.04 & & & & \\
\hline & Sociabilidade/empatia & -.038 & .97 & -.005 & & & & \\
\hline & Flow (DFS-2) & & & & & & & \\
\hline & Fusão entre ação e consciência & -.85 & .40 & -.070 & & & & \\
\hline & Experiência autotélica & 1.65 & .10 & .18 & & & & \\
\hline & $\begin{array}{l}\text { Balanço entre desafio e } \\
\text { competência }\end{array}$ & 2.26 & .025 & .27 & & & & \\
\hline & Clareza de objetivos & .14 & .89 & .012 & & & & \\
\hline & Concentração na tarefa & -.078 & .94 & -.007 & & & & \\
\hline & Perda de autoconsciência & -1.64 & .10 & -.11 & & & & \\
\hline & Perceção de controlo & -.24 & .81 & -.025 & & & & \\
\hline & $\begin{array}{l}\text { Transformação do sentido de } \\
\text { tempo }\end{array}$ & -.44 & .66 & -.029 & & & & \\
\hline & Feedback rigoroso & 1.40 & .16 & .16 & & & & \\
\hline
\end{tabular}

Nota. Os valores $p$ a negrito são estatisticamente significativos.

A perceção subjetiva do estudante sobre o seu desempenho académico foi essencialmente predita pela perceção de balanço equilibrado entre o grau de desafio e a sua competência, uma das dimensões do flow. O estudante constrói a sua perceção de competência considerando o nível de exigência ou desafio e de complexidade ou dificuldade da tarefa que lhe é apresentada. Neste sentido, o equilíbrio no grau de complexidade das tarefas e matérias apresentadas aos estudantes tende a influenciar positivamente a perceção de competência dos mesmos, na medida em que evita o surgimento de ansiedade naqueles que não conseguem cumprir os objetivos dos conteúdos, ou de aborrecimento naqueles que facilmente atingem metas pouco complexas (Nakamura \& Csikszentmihalyi, 2002).

A explicação do desempenho académico objetivo do estudante foi, neste estudo, parcialmente realizada pelas variáveis positivas em análise (13\% $R^{2}$ ajustado), nomeadamente pela experiência autotélica de flow e pelas dimensões de auto-estima e bem-estar físico da felicidade. Considera-se como autotélica uma experiência cuja realização cumpre um fim em si mesmo, isto é, o indivíduo sente-se intrinsecamente recompensado pela experiência, sem considerar os possíveis resultados que daí possam advir (Csikszentmihalyi, 1990). Em particular, no caso dos estudantes autotélicos, que valorizam o processo educativo e cuja motivação para a aprendizagem é eminentemente intrínseca, ao investirem e apreciarem a experiência de aprendizagem conseguem potenciar a obtenção de resultados objetivos concretos. Por outro lado, a autoestima do estudante poderá ter um efeito positivo sobre o seu desempenho académico, permitindo reforçar comportamentos adaptativos neste domínio. Ainda, os níveis de envolvimento e comprometimento do estudante estarão associados à sua maior capacidade de concentração e trabalho académico. Neste estudo, contrariamente à literatura (Bukhari \& Khanam, 2016; Costa \& Faria, 2014, 2015; O’Boyle et al., 2011; Perera, 2016), a IE não demonstrou capacidade de predizer o rendimento académico dos estudantes.

Este estudo não está isento de limitações. De facto, sendo um estudo de desenho transversal não 
Quadro 4. Modelos de regressão linear de variáveis positivas sobre o bem-estar subjetivo

\begin{tabular}{|c|c|c|c|c|c|c|c|c|}
\hline Modelo & & $t$ & $p$ & $\beta$ & $F$ & $g l$ & $p$ & $\begin{array}{c}R^{2} \\
\text { ajustado }\end{array}$ \\
\hline \multirow{24}{*}{$\begin{array}{l}\text { Bem- } \\
\text { estar } \\
\text { subjetivo }\end{array}$} & & & & & 16.60 & 20 & .001 & .59 \\
\hline & $\begin{array}{l}\text { Inteligência emocional (TEIQue- } \\
\text { SF) }\end{array}$ & & & & & & & \\
\hline & Emocionalidade & -.13 & .90 & -.008 & & & & \\
\hline & Sociabilidade & -.067 & .95 & -.004 & & & & \\
\hline & Autocontrolo & 2.46 & .015 & .14 & & & & \\
\hline & Bem-estar & .97 & .34 & .085 & & & & \\
\hline & Felicidade (OHI) & & & & & & & \\
\hline & Alegria & -1.32 & .19 & -.092 & & & & \\
\hline & Eficácia pessoal & .51 & .61 & .041 & & & & \\
\hline & Bem-estar físico & .16 & .87 & .013 & & & & \\
\hline & Perspetiva positiva & 1.35 & .18 & .11 & & & & \\
\hline & Satisfação com a vida & 1.46 & .15 & .11 & & & & \\
\hline & Autoestima & -1.19 & .24 & -.074 & & & & \\
\hline & Sociabilidade/empatia & -1.54 & .13 & -.15 & & & & \\
\hline & Flow (DFS-2) & & & & & & & \\
\hline & Fusão entre ação e consciência & -2.98 & .003 & -.19 & & & & \\
\hline & Experiência autotélica & 7.90 & .001 & .65 & & & & \\
\hline & Balanço entre desafio e competência & 1.06 & .29 & .094 & & & & \\
\hline & Clareza de objetivos & -.61 & .54 & -.042 & & & & \\
\hline & Concentração na tarefa & -1.17 & .24 & -.081 & & & & \\
\hline & Perda de autoconsciência & 2.28 & .024 & .11 & & & & \\
\hline & Perceção de controlo & 1.51 & .13 & .12 & & & & \\
\hline & Transformação do sentido de tempo & -.59 & .56 & -.029 & & & & \\
\hline & Feedback rigoroso & .54 & .59 & .045 & & & & \\
\hline
\end{tabular}

Nota. Os valores $p$ a negrito são estatisticamente significativos.

permitiu explorar relações de causalidade entre as variáveis analisadas e os respetivos desempenhos académicos e bem-estar subjetivo. $\mathrm{O}$ facto de a amostra ser maioritariamente oriunda de cursos universitários de áreas de ciências sociais limita a possibilidade de generalização dos resultados a outras áreas, nomeadamente às de ciências exatas, onde as variáveis positivas podem ter um impacto diferente sobre o desempenho dos indivíduos. Mais ainda, a menor percentagem de trabalhadores-estudantes e de finalistas reduziu a possibilidade de explorar o impacto destas variáveis em estudantes com um perfil diferenciado, já com responsabilidades acrescidas no mundo profissional. $\mathrm{O}$ uso de medidas genéricas de IE e de felicidade, não específicas do contexto académico, poderá ter limitado a sua capacidade preditiva sobre os desempenhos académicos. Estudos futuros deverão confirmar e aprofundar os resultados encontrados neste estudo, explorando a relação entre as variáveis em diferentes amostras de estudantes e ou profissionais e em diferentes contextos existenciais.
Uma vez que, neste estudo, as variáveis positivas analisadas demonstraram, globalmente, tendência para influenciar positivamente o bemestar e os resultados académicos do aluno, tornase importante valorizar e potenciar o desenvolvimento de competências socioemocionais e interpessoais dos estudantes em contexto académico. Neste sentido, poderá ser relevante a promoção de atividades que possibilitem aos alunos uma maior aproximação e partilha com os seus pares, maior adaptação e envolvimento com o contexto académico, e que fomentem o desenvolvimento das suas competências socioemocionais e interpessoais, como por exemplo: uso de estratégias formais e informais em sala de aula, que promovam o trabalho colaborativo, feedback positivo, criatividade, estratégias de comunicação e resolução de problemas; oferta de atividades extracurriculares (e.g., desporto, associativismo, clubes, artes, voluntariado); promoção de um acompanhamento individualizado do estudante ao longo do seu percurso académico, através da 
oferta de atividades que fomentem o desenvolvimento pessoal e socioemocional (e.g., tutorias e mentoria, entre outros); oferta de ações de formação no âmbito do desenvolvimento de competências socioemocionais.

Este trabalho reforça a importância que a promoção de dimensões socioemocionais da competência pode assumir na definição de objetivos e na obtenção de resultados académicos otimizados, como importante contributo para a promoção da sua adaptação aos contextos universitários e para a sua preparação para os desafios futuros do contexto profissional.

\section{Referências}

Allen, M., \& McCarthy, P. J. (2016). Be happy in your work: The role of positive psychology in working with change and performance. Journal of Change Management, 16(1), 5574. doi:10.1080/14697017.2015.1128471

Antaramian, S. (2015). Assessing psychological symptoms and well-being: Application of a dual-factor mental health model to understand college student performance. Journal of Psychoeducational Assessment, 33(5), 419429. https://doi.org/10.1177/0734282914557727

Argyle, M., Martin, M., \& Crossland, J. (1989). Happiness as a function of personality and social encounters. In P. Forgas \& J. M. Innes (Eds.), Recent advances in social psychology: an international perspective (pp. 189-203). Amsterdam: North Holland, Elsevier Science.

Asakawa, K. (2004). Flow experience and autotelic personality in Japanese college students: How do they experience challenges in daily life? Journal of Happiness Studies, 5, 123-154. doi:10.1023/B:JOHS.0000035915.97836.89

Bakker, A. B. (2005). Flow among music teachers and their students: The crossover of peak experiences. Journal of Vocational Behavior, 66(1), 26-44. doi:10.1016/j.jvb.2003.11.001

Barrick, M. R., Stewart, G. L., \& Piotrowski, M. (2002). Personality and job performance: Test of the mediating effects of motivation among sales representatives. Journal of Applied Psychology, 87, 4351. doi:10.1037/0021-9010.87.1.43
Bukhari, S. R., \& Khanam, S. J. (2016). Trait emotional intelligence as a predictor of academic performance in university students. Pakistan Journal of Psychology, 47(2), 33-44. doi:10.1111/j.1365-2702.2012.04199.x.

Bukhari, S. R., \& Khanam, S. J. (2017). Relationship of academic performance and well-being in university students. Pakistan Journal of Medical Research, 56(4), 126-130.

Carvalho, D., Neto, F., \& Mavroveli, S. (2010). Trait emotional intelligence and disposition for forgiveness. Psychological Reports, 107(2), 526534. doi:10.2466/02.09.20.21.PR0.107.5.526-534

Costa, A., \& Faria, L. (2014). Avaliação da inteligência emocional: A relação entre medidas de desempenho e de autorrelato. Psicologia: Teoria e Pesquisa, 30(3), 339$346 . \quad$ http://dx.doi.org/10.1590/S010237722014000300011

Costa, A., \& Faria, L. (2015). The impact of emotional intelligence on academic achievement: A longitudinal study in Portuguese secondary school. Learning and Individual Differences, 37, 38-47. https://doi.org/10.1016/j.lindif.2014.11.011

Csikszentmihalyi, M. (1990). Flow. The psychology of optimal experience (1st ed.). New York: Harper \& Row.

Delle Fave, A. (2013). Past, present, and future of flow. In S. A. David, I. Boniwell, \& A. C. Ayers (Eds.), The Oxford Handbook of Happiness. Oxford, UK: Oxford University Press. doi:10.1093/oxfordhb/9780199557257.013.0005

Di Fabio, A., \& Kenny, M. E. (2016). Promoting well-being: The contribution of emotional intelligence. Frontiers in Psychology, 7(1182), 1-13. doi:10.3389/fpsyg.2016.01182

Diener, E. (1984). Subjective well-being. Psychological Bulletin, 95, 542-575.

Diener, E., Suh, E., \& Oishi, S. (1997). Recent findings on subjective well-being. Indian Journal of Clinical Psychology, 24(1), 25-41. doi:10.1037/0033-2909.125.2.276

Erez, A., \& Judge, A. (2001). Relationship of core self-evaluations to goal setting, motivation, and performance. Journal of Applied Psychology, $\quad$ 86, 1270-1279. doi:10.1037/0021-9010.86.6.1270

Ferreira Novo, R. (2005). Bem-estar e psicologia: Conceitos e propostas de avaliação. Revista 
Iberoamericana de Diagnóstico y Evaluación - e Avaliação Psicológica, 2(20), 183-203.

Figueira, C., Marques Pinto, A., Lima, L., Matos, A. P., \& Cherpe, S. (2014). Adapting the Mental Health Continuum - LF - for adults in Portuguese university students. Revista Iberoamericana de Diagnóstico y Evaluación - e Avaliação Psicológica, 2(38), 93-116.

Garg, R., Levin, E. \& Tremblay, L. (2016). Emotional intelligence: Impact on postsecondary academic achievement. Social Psychology of Education, 19(3), 627-642. doi:10.1007/s11218-016-9338-x

Gouveia, M. J., Pais-Ribeiro, J. L., Marques, M. M., \& Carvalho, C. M. (2012). Validity and reliability of the Portuguese version of the Dispositional Flow Scale-2 in exercise. Revista de Psicología Del Deporte, 21(1), 8188.

Gouveia, V. V., Fonsêca, P. N., Lins, S. L. B., Lima, A. V., \& Gouveia, R. S. V. (2008). Escala de Bem-Estar Afetivo no Trabalho (JAWS): Evidências de validade fatorial e consistência interna. Psicologia: Reflexão $e$ Crítica, 21(3), 464-473. http://dx.doi.org/10.1590/S010279722008000300015

Hurtz, G. M., \& Donovan, J. J. (2000). Personality and job performance: The Big Five revisited. Journal of Applied Psychology, 85, 869-879. doi:10.1037/0021-9010.85.6.869

Ilies, R., Wagner, D., Wilson, K., Ceja, L., Johnson, M., DeRue, S., \& Ilgen, D. (2017). Flow at work and basic psychological needs: Effects on well-being. Applied Psychology, 66(1), 3-24. doi:10.1111/apps.12075

Jackson, S. A., \& Eklund, R. C. (2002). Assessing flow in physical activity: The Flow State Scale-2 and Dispositional Flow Scale-2. Journal of Sport \& Exercise Psychology, 24, 133-150. doi:10.1123/jsep.24.2.133

Jackson, S. A., Ford, S. K., Kimiecik, J. C., \& Marsh, H. W. (1998). Psychological correlates of flow in sport. Journal of Sport and Exercise Psychology, 20(4), 358-378. doi:10.1123/jsep.20.4.358

Jackson, S. A., \& Marsh, H. W. (1996). Development and validation of a scale to measure optimal experience: The Flow State Scale. Journal of Sport and Exercise
Psychology, 18(1),

17-35.

doi: 10.1123/jsep.18.1.17

Jalali, Z., \& Heidari, A. (2016). The relationship between happiness, subjective well-being, creativity and job performance of primary school teachers in Ramhormoz city. International Education Studies, 9(6), 45-52. http://dx.doi.org/10.5539/ies.v9n6p45

Joo, Y. J., Oh, E., \& Kim, S. M. (2015). Motivation, instructional design, flow, and academic achievement at a Korean online university: A structural equation modeling study. Journal of Computing in Higher Education, 27(1), 28-46.

doi:10.1007/s12528-015-9090-9

Joseph, D. L., Jin, J., Newman, D. A., and O’Boyle, E. H. (2015). Why does selfreported emotional intelligence predict job performance? A meta-analytic investigation of mixed EI. Journal of Applied Psychology, 100(2), 298-342. doi:10.1037/a0037681

Joshanloo, M. (2016). Revisiting the empirical distinction between hedonic and eudaimonic aspects of well-being using exploratory structural equation modeling. Journal of Happiness Studies, 17(5), 2023-2036.

doi:10.1007/s10902-015-9683-z

Katwyk, P. T., Fox, S., Spector, P. E., \& Kelloway, E. K. (2000). Using the JobRelated Affective Well-Being Scale (JAWS) to investigate affective responses to work stressors. Journal of Occupational Health Psychology, 5(2), 219-230. doi: 10.1037//1076-8998.5.2.219

Koydemir, S., \& Schütz, A. (2012). Emotional intelligence predicts components of subjective well-being beyond personality: A two-country study using self- and informant reports. The Journal of Positive Psychology, 7(2), 107118. doi:10.1080/17439760.2011.647050

Kuo, T., \& Ho, L. (2010). Individual difference and job performance: The relationships among personal factors, job characteristics, flow experience, and service quality. Social Behavior and Personality, 38(4), 531-552. doi:10.2224/sbp.2010.38.4.531

Linley, P. A., Joseph, S., Harrington, S., \& Wood, A. M. (2006). Positive psychology: Past, present, and (possible) future. The Journal of 
Positive Psychology, 1(1), 3-16. http://dx.doi.org/10.1080/17439760500372796

Lyubomirsky, S. (2008). The how of happiness: A new approach to getting the life you want. New York, USA: The Penguin Press.

Lyubomirsky, S., King, L., \& Diener, E. (2005). The benefits of frequent positive affect: Does happiness lead to success? Psychological Bulletin, 131(6), 803-855. doi:10.1037/0033-2909.131.6.803

Man, M., \& Ticu, C. (2015). Subjective WellBeing and professional Performance. Revista Academiei Fortelor Terestre, 20(2), 211-216.

Matthewman, L., Jodhan-Gall, D., Nowlan, J., OSullivan, N., \& Patel, Z. (2018). Primed, prepped and primped: Reflections on enhancing student well-being in tertiary education. Psychology Teaching Review, 24(1), 67-76.

Mayer, J. D., \& Salovey, P. (1993). The intelligence of emotional intelligence. Intelligence, 17, 433-442. doi:10.1016/0160-2896(93)90010-3

Nakamura, J., \& Csikszentmihalyi, M. (2002). The concept of flow. The Handbook of Positive Psychology, 13, 89-105. doi:10.1007/978-94-017-9088-8_16

Neto, F. (2001). Personality predictors of happiness. Psychological Reports, 88, 817824. doi:10.2466/pr0.2001.88.3.817

O’Boyle, E. H., Humphrey, R. H., Pollack, J. M., Hawver, T. H., \& Story, P. A. (2011). The relation between emotional intelligence and job performance: A meta-analysis. Journal of Organizational Behavior, 32, 788-818. doi:10.1002/job.714

Perera, H. N. (2015). The internal structure of responses to the trait emotional intelligence questionnaire-short form: An exploratory structural equation modeling approach. Journal of Personality Assessment, 97(4), 411423. doi:10.1080/00223891.2015.1014042

Perera, H. N. (2016). The role of trait emotional intelligence in academic performance: Theoretical overview and empirical update. The Journal of Psychology, 150(2), 227-249. doi:10.1080/00223980.2015.1079161

Petrides, K. V., \& Furnham, A. (2001). Trait emotional intelligence: Psychometric investigation with reference to established trait taxonomies. European Journal of Personality, 15(6), 425-448. doi: 10.1002/per.416

Petrides, K. V., \& Furnham, A. (2006). The role of trait emotional intelligence in a gender-specific model of organizational variables. Journal of Applied Social Psychology, 36(2), 552-569. https://doi.org/10.1111/j.0021-9029.2006.00019.x

Rich, B. L., LePine, J. A., \& Crawford, E. R. (2010). Job engagement: Antecedents and effects on job performance. Academy of Management Journal, 53, 617-635. doi:10.5465/AMJ.2010.51468988

Ryan, R. M., \& Deci, E. L. (2001). On happiness and human potentials: A review of research on hedonic and eudaimonic well-being. Annual Review of Psychology, 52(1), 141-166. doi:10.1146/annurev.psych.52.1.141

Sánchez-Álvarez, N., Extremera, N., \& Fernández-Berrocal, P. (2016). The relation between emotional intelligence and subjective well-being: A meta-analytic investigation. Journal of Positive Psychology, 11(3), 276285. doi:10.1080/17439760.2015.1058968

Seligman, M. E. P. (2011). Flourish: A visionary new understanding of happiness and wellbeing. New York, NY, US: Free Press.

Silveira, C., Norton, A., Brandão, I., \& RomaTorres, A. (2011). Saúde mental em estudantes do ensino superior: Experiência da consulta de Psiquiatria do Centro Hospitalar São João. Acta Médica Portuguesa, 24(S2), 247-256. https://doi.org/22849909

Steele, J., \& Fullagar, C. (2009). Facilitators and outcomes of student engagement in a college setting. Journal of Psychology: Interdisciplinary and Applied, 143(1), 5-27. doi:10.3200/JRLP.143.1.5-27

Sumaya, I. C., \& Darling, E. (2018). Procrastination, flow, and academic performance in real time using the experience sampling method. The Journal of Genetic Psychology Research, 179(3), 123-131. doi:10.1080/00221325.2018.1449097

Wang, M., Zou, H., Zhang, W., \& Hou, K. (2018). Emotional intelligence and subjective wellbeing in Chinese university students: The role of humor. Journal of Happiness Studies. doi:10.1007/s10902-018-9982-2 
Warr, P. (1987). Work, unemployment, and mental health. Oxford, England: Oxford University Press.

Warr, P. (1990). The measurement of well-being and other aspects of mental health. Journal of Occupational Psychology, 63(3), 193-210. doi:10.1111/j.2044-8325.1990.tb00521.x

Youssef, C. M., \& Luthans, F. (2007). Positive organizational behavior in the workplace: The impact of hope, optimism, and resilience. Journal of Management, 33(5), 774-800. doi:10.1177/0149206307305562

Zeidner, M., Matthews, G., \& Roberts, R. D. (2012). The emotional intelligence, health, and well-being nexus: What have we learned and what have we missed? Applied Psychology: Health and Well-Being, 4, 1-30. doi: 10.1111/j.1758-0854.2011.01062.x 